\title{
Buckling of 2D nano hetero-structures with moire patterns
}

\author{
Y. Chandra ${ }^{\mathrm{a}}$, E.I. Saavedra Flores ${ }^{*, \mathrm{~b}}$, S. Adhikari ${ }^{\mathrm{a}}$ \\ ${ }^{a}$ Zienkiewicz Centre for Computational Engineering, Swansea University, Swansea SA1 8EN, UK \\ ${ }^{b}$ Departamento de Ingeniería en Obras Civiles, Universidad de Santiago de Chile, Av. Ecuador 3659, Estación \\ Central, Santiago, Chile
}

\begin{abstract}
Moire pattern arises from the lattice mismatch between two different nanosheets. The discovery of the Moire pattern has resulted in breakthrough properties in 2D carbon-based nanostructures such as graphene. Here we investigate the impact of a Moire pattern on mechanical properties of bi-layer 2D nanosheets. In particular, buckling instability of 2D carbonbased nano hetero-structures is investigated using atomistic finite element approaches. Nano hetero-structures considered are graphene-hBN (hexagonal Boron Nitride) and graphene-MoS ${ }_{2}$ (Molybdenum disulphide). Bilayer graphene has also been considered in the buckling analysis, by orienting the individual sheets at moire angle. Atomistic simulation methodology uses elastic beams to represent intra-sheet atomic bonds and elastic springs to represent inter-sheet atomic interactions. The influence of different boundary conditions and sheet length on the buckling of nano hetero-structures has been investigated. The bridged nano hetero-structures are found be displaying higher buckling strength as compared to cantilever sheets.
\end{abstract}

Key words: Buckling instability; nano hetero-structures; graphene; hexagonal Boron Nitride; Molybdenum Disulphide; moire angle.

\section{Introduction}

Since the revolutionary discovery of graphene in 2004 [1], a progressively increasing interest by $2 \mathrm{D}$ nanomaterials has been observed within scientific and engineering communities. Currently, various types of $2 \mathrm{D}$ nanomaterials are under investigation in order to exploit their extraordinary potential as the next generation of super materials with remarkable physical properties. For instance, graphene shows great buckling strength [2, 3], hexogonal Boron Nitride (hBN) [4] possesses outstanding spin polarized states [5] and Molybdenum Disulphide $\left(\mathrm{MoS}_{2}\right)$ [6] offers ex-

\footnotetext{
${ }^{*}$ Corresponding author. Tel: +56 (2) 27182803

Email address: erick.saavedra@usach.cl (E.I. Saavedra Flores)
} 
ceptional electrical transport properties [7]. If different 2D nanomaterials are combined into one single nano hetero-structure, all these properties can be harnessed. Such hetero-structures are also referred to as van der Waals hetero-structures. The lattice mismatch that occurs between the lattices of nanosheets within the van der Waals hetero-structures leads to unique properties [8]. Similar lattice mismatch has also been observed in multilayer graphene sheets twisted at moire angles [9]. Such moire patterns in twisted bilayer graphene, overlaid graphene-hBN and overlaid graphene-MoS 2 are shown in Fig. 1. The relevance of moire angles between offset layers of different atoms lies in the fact that they produce changes in their electrical properties, which can result in the synthesis of new materials with potentially tailored properties. For instance, it has been found that graphene turns superconducting when two stacked graphene layers rotate by an angle of $1.1^{\circ}$, which represents an example of how atomically thin materials can produce completely new electrical properties [10].

Recent advances in this area, worth mentioning are the references[11-13]. Nika et al[11] studied specific heat of twisted bilayer graphene at an angle of $21.8^{\circ}$, and compared its performance against that of graphite and untwisted bilayer graphene. This article concluded that the phonon specific heat is highly dependent on a twist angle under low-temperature conditions. This means phonon engineering of thermal properties of layered materials is possible by twisting the basal atomic planes. Especially, the authors suggested that the specific heat of bilayer graphene can decrease by up to $15 \%$, when a twist angle is introduced at a temperature of $1 \mathrm{~K}$. Mortazavi et al[12] performed mechanical analysis of a nano hetero-structure consisting of a single layer $\mathrm{MoTe}_{2}$. This nanomaterial $\mathrm{MoTe}_{2}$ belong to the class of transition metal dichalcogenides. These authors[12] quantified elastic modulus, Poisson's ratio, strain at the ultimate tensile strength point and the ultimate tensile strength of $\mathrm{MoTe}_{2}$ under various atomic configurations, with the aid of first-principles density functional theory. These authors[12] suggested anisotropic mechanical properties, bandgap under tension, auxetic Poisson's ratio and remarkable tensile strength, for $\mathrm{MoTe}_{2}$. Vargas et al[13] studied thermal and electrical transport in a polycrystalline graphene-hBN hetero-structure, with the aid of tight-binding simulations and combined molecular dynamics-finite element simulations. This study showed a significant influence of hBN content on the thermal conductivity of the graphene-hBN hetero-structure. Furthermore, this study concluded that the graphene-hBN hetero-structure could be a conductor or a semiconductor, depending on the content of hBN

In the present work, a new contribution is made in order to provide further insight into the 
linear buckling of bilayer graphene sheets with sheet alignment at a moire angle of $1.1^{\circ}$, and graphene-hBN and graphene- $\mathrm{MoS}_{2}$ bilayer sheets. The isometric views of graphene-graphene, graphene-hBN and graphene- $\mathrm{MoS}_{2}$ bilayer systems are shown in Fig. 2. In this paper, we compute the critical buckling loads by means of a finite element (FE) based lattice approach. This numerical strategy establishes a linkage between the actual atomistic system and an equivalent mechanical model at the atomic bond level and provides a way to capture the atomistic response by means of conventional FE analyses and classical beam elements. Furthermore, we investigate the influence of boundary conditions and lengths of nanosheets on their buckling capacity by means of a detailed set of numerical experiments. We must note that nonlinear buckling analyses on carbon-based nanostructures have been performed in the past $[3,14]$, however, such simulations are complex and computationally demanding, and are normally justified when a post-buckling response is investigated. At present, we find it more convenient to perform linear simulations in view of our interest in the eigenvalues and eigenvectors of the nanosheet configurations studied here.

The paper is organized as follows. Section 2 presents the description of the equivalent mechanical model of nano hetero-structures by means of the finite element method (FEM). The results and discussions of the present work are given in Section 3. Finally, Section 4 summarizes our main conclusions.

\section{Atomistic FE models of nano hetero-structures using FEM}

The atomistic models deployed here are based on the FE methodologies developed by the authors to study graphene and its associated nano structures [2, 3, 15-18]. In this research work the FE analysis tool OPTISTRUCT has been used to model the dynamic behaviour of nano hetero-structures. The covalent bonds are represented by equivalent 3D Timoshenko FE beams and the atoms are represented by FE nodes. Within OPTISTRUCT, the element type CBEAM has been used to represent beams. The diameter and the Young's modulus of the beam elements are computed by using the following equations of force constants $K_{r}$ and $k_{\theta}$ shown below:

$$
\begin{gathered}
K_{r}=\frac{E A}{L}, \text { and } \\
K_{\theta}=\frac{E I}{L} .
\end{gathered}
$$




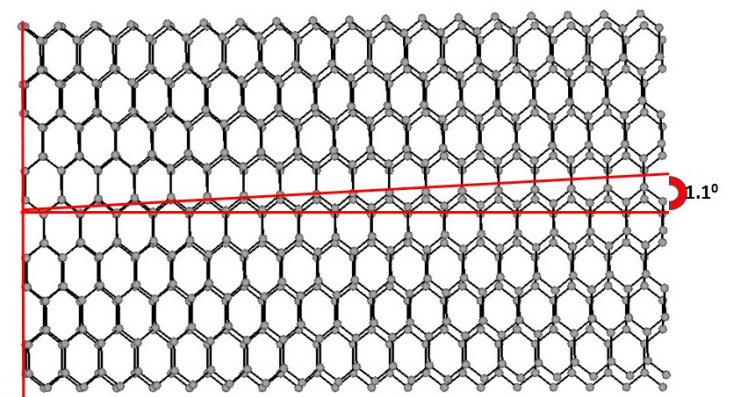

(a) Mismatch in bilayer graphene introduced by twisting at an angle of $1.1^{\circ}$

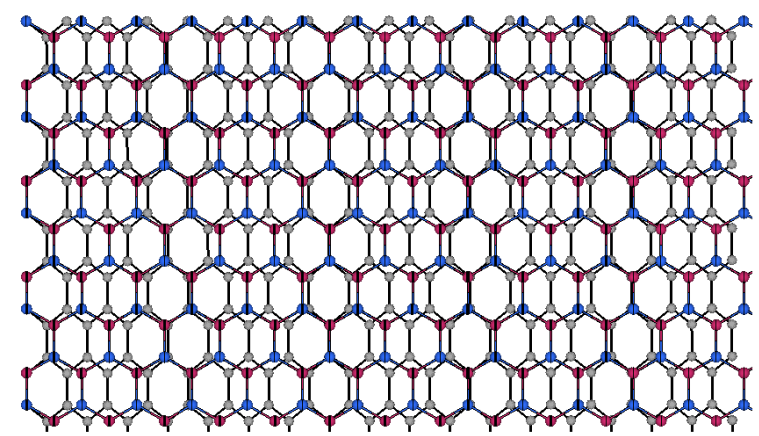

(b) Lattice mismatch in graphene-hBN bilayer sheet leading to moire pattern

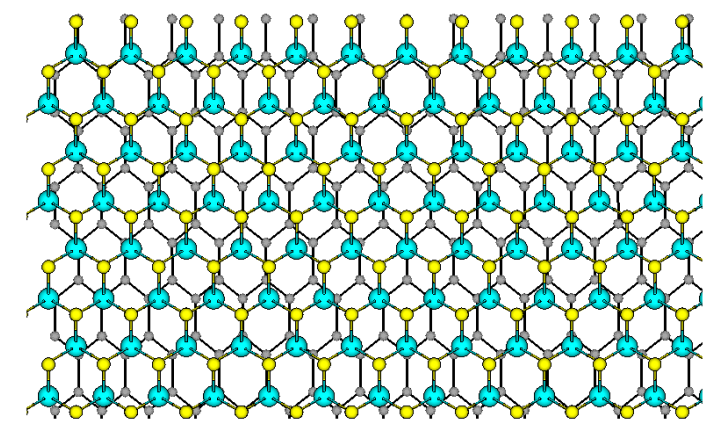

(c) Lattice mismatch in graphene-MoS 2 bilayer sheet leading to moire pattern

Figure 1: Lattice mismatch in overlapped nanosheets leading to a moire pattern.

In the above equation, $E$ is the Young's modulus of the beams, $I$ is the area moment of inertia of the beams, $A$ is the cross section of the beams and $L$ is the length of the beams. The above two equations are derived from stretching and bending interatomic potentials $[19,20]$. The numerical values of force constants $K_{r}$ and $K_{\theta}$ for atomic interactions such as C-C, B-N and Mo-S are available in the literature [19,20]. By substituting these values in Eq. 1 and Eq. 2, essential parameters to model covalent bond beams, such as beam diameter $d$ and beam Young's modulus $E$ can be calculated.

The equivalent axial force for a L-J potential between a pair of atoms $i$ and $j$ belonging to different nanosheets can be defined as [21]

$$
F_{i j}=\frac{\partial V_{i j}}{\partial r}
$$

where, $r$ is the atomic displacement between $i$ and $j$ (layer-layer length). As per Girifalco et al. 
[22], the force between the atoms $i$ and $j$ can also be represented by

$$
F_{i j}=-12 \epsilon\left[\left(\frac{r_{\min }}{y}\right)^{13}-\left(\frac{r_{\min }}{y}\right)^{7}\right],
$$

where, $y=r_{\min }+\delta r, \delta r$ is the atomic displacement along the length $i j$. The $r_{\min }$ (in $\AA$ ) is given by $2^{\frac{1}{6}} \sigma$, with $\sigma=(A / B)^{1 / 6}$. The $B$ and $A$ are attractive and repulsive constants, respectively. In the current research work, three different nanosheets have been considered, namely graphene, $\mathrm{hBN}$ and $\mathrm{MoS}_{2}$. Hetero-structures of graphene-hBN and graphene-MoS $\mathrm{M}_{2}$ have been studied under buckling loads. These hetero-structures lead to C-C,C-B,C-N,C-M and C-S interlayer atomic interactions, where $\mathrm{C}, \mathrm{B}, \mathrm{N}, \mathrm{M}$ and $\mathrm{S}$ are carbon, boron, nitrogen, molybdenum and sulphide atoms, respectively. The values of $\sigma$ and $\epsilon$ for each individual van der Waals atomic interactions are obtained from various references [23-26]. In the atomistic FE models, we have used spring elements to form a connection between two layers of the bilayer structure representing L-J interactions. The force deflection curve for L-J springs has been calculated by using the relation in Eq. 4. Within the FE analysis tool OPTISTRUCT, the L-J springs of interlayer interactions are modeled by the element type CBUSH and by using the curves of Eq. 4 as input properties.

\subsection{Validity of atomistic models}

The idea of performing atomistic simulations using the finite element method has evolved since 2003 [20]. Deriving equivalent mechanical properties of atomic interactions using harmonic potentials $\left(K_{r}\right.$ and $\left.K_{\theta}\right)$ is a well established concept $[27,28]$. Such an approach will establish near-perfect equivalence between molecular mechanics and nanostructure mechanics. Furthermore, in such simulations, the most important bond deformation modes such as bond stretching and angle variations are accurately captured. The current authors have validated the finite element approach of atomistic simulations against analytical models [15, 16], experimental observations[17] and also molecular dynamic simulations[18]. The accuracy of the atomistic finite element model is found to be as high as $3.8 \%$ in the case of nanosheets and $5 \%$ in the case of nanocomposites. Furthermore, the results of finite element based atomistic simulation are found to close to experimental results with a very low margin difference (1.8\%). It is important to note that the prediction of buckling strength of nanostructures such as single-layer graphene, bilayer graphene, single-wall carbon nanotube, and nanocomposites, using finite element based atomistic method by current authors $[2,3,14]$ has been widely accepted by the nanomaterials research community. The current research involves applications of same well established 
method $[2,3,14]$ to explore the buckling characteristics of nano hetero-structures. The current research work can serve as a benchmark for researchers intending to introduce high fidelity into the buckling models of nano hetero-structures, in the future.

We must note that in the present FE modelling approach, an explicit expression for a potential energy function is required in order to establish an equivalence between the mechanical and atomic bond energies. Such bonds can stretch, rotate or twist between neighbouring atoms or molecules and eventually, produce a global deformation response. However, the modelling of re-formed molecular bonds due to chemical reactions is a challenging task of difficult implementation within the present computational simulation framework. Some examples of these chemical reactions can be found in pyrolysis and combustion of hydrocarbon systems where large molecules breakdown into smaller molecules in the presence of heat. For the modelling of such complex reactions, the use of reactive force-field such as ReaxFF [29] is recommended, particularly when new bonds are chemically generated. We remark that the present FE approach is more suitable to capture the physical deformation process of atoms or molecules instead of describing the chemical reaction kinetics of atomic systems.

\subsection{Stability of nanosheets}

In the Moire pattern of 2-dimensional multi-layered nanosheets, it is a challenging task to keep an inter sheet angle without fixing the system. Therefore, the sheets tend to re-rotate to the original positions, when a boundary fixing is absent. However, in the current work, the sheets have been placed at an angle of $1.1^{\circ}$ against each other, before the analysis is performed. Furthermore, in the numerical models of nanosheets, stability is ensured due to the enforcement of mechanical constraints in all six degrees of freedom, as shown in 3(a) and 4(a). It is also important to note that the relevant works[11, 30-32] did not consider the issue of angular stability of the twisted sheets. Furthermore, Cao et al[33] manually rotated individual sheets of bilayer graphene at an angle higher than the magic angle but did not report the issue of angular stability. Although, various types of interlayer interactions are possible between individual sheets, including van der Waals, covalent, part covalent, electrostatic(Columb), ionic and $\pi-$ $\pi$. Only van der Waals interactions modelled by LJ-potentials are found to be effective in representing interlayer force transfer while calculating structural properties of multi-layered nanosheets[2, 15, 21, 22]. 


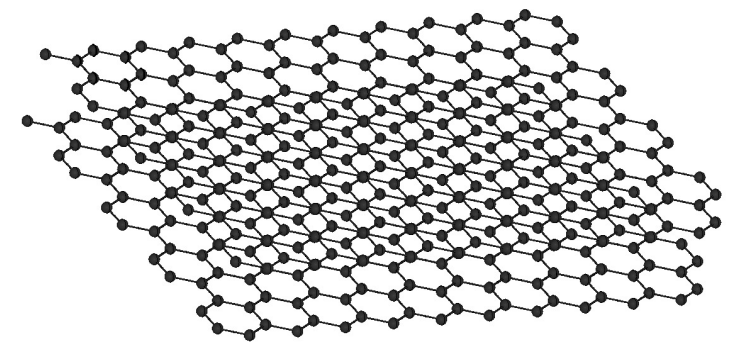

(a) Bilayer graphene sheet

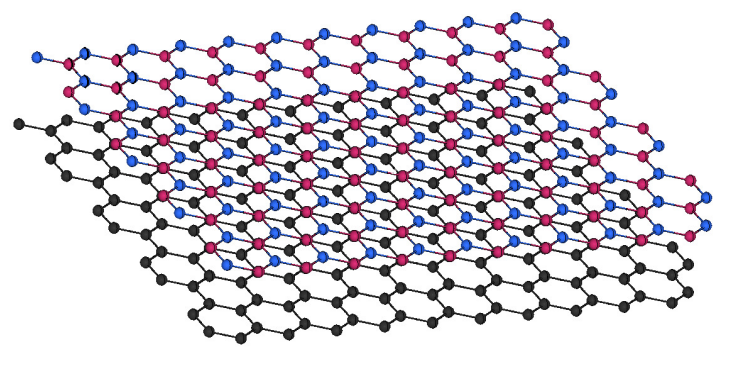

(b) Graphene-hBN hetero-structure

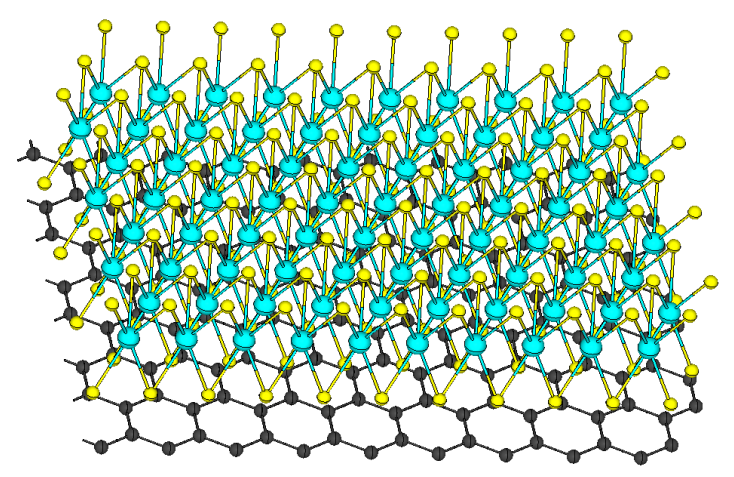

(c) Graphene-Mos 2 hetero-structure

Figure 2: Isometric views of bilayer graphene, graphene-hBN and graphene- $\mathrm{MoS}_{2}$ sheets.

\section{Results and discussions}

As the buckling capacity of nanosheets is greatly influenced by their boundary conditions, we investigate here the eigenvalues and eigenvectors of cantilevered and bridged nanosheets. The variation of the aspect ratio by changing the length is also studied. The cantilever boundary condition involves constraining all the degrees of freedom located at one edge of the sheet (Fig. 3(a)). Bridged boundary condition involves constraining all the degrees of freedom at two opposite edges (Fig. 4(a)). The two layer nanosheet structure undergoes buckling when a unit compressive load is applied at opposite sides (Refer to Fig. 3(a) and Fig. 4(a)). Buckling analysis has been performed using the atomistic finite element approach explained in the previous section. The solution process within the FE code OPTISTRUCT involves the generation of a geometric stiffness matrix based on a unit force in the direction of buckling, followed by the numerical prediction of the eigenvalues and eigenvectors by means of a standard Lanczos solver. The buckling mode shapes for nanosheets under cantilevered boundary conditions are shown in Fig. 3(b), Fig. 3(c) and Fig. 3(d) for bilayer graphene, graphene-hBN and graphene- $\mathrm{MoS}_{2}$, respectively. The buckling mode shapes for nanosheets under bridged boundary conditions for 
bilayer graphene, graphene-hBN and graphene- $\mathrm{MoS}_{2}$ are shown in Fig. 4(b), Fig. 4(c) and Fig. 4(d), respectively. These buckling mode shapes for double layer systems are found to be identical to those of single layer graphene (Figure not shown here).

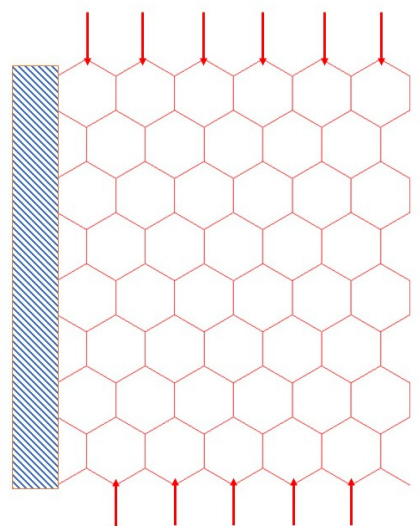

(a) Cantilever boundary condition

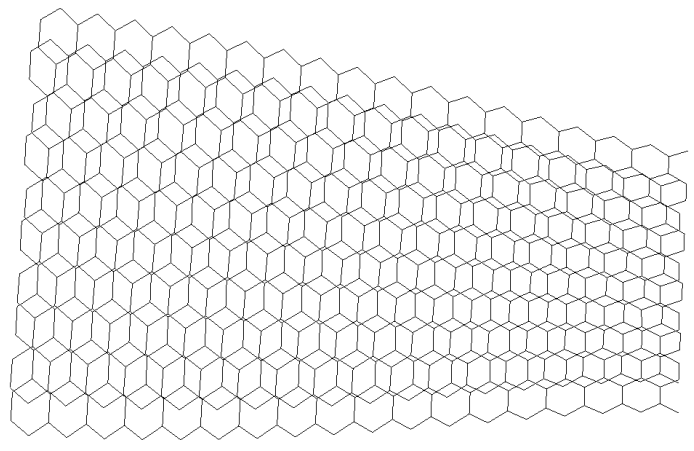

(b) First buckling mode shape of bilayer graphene

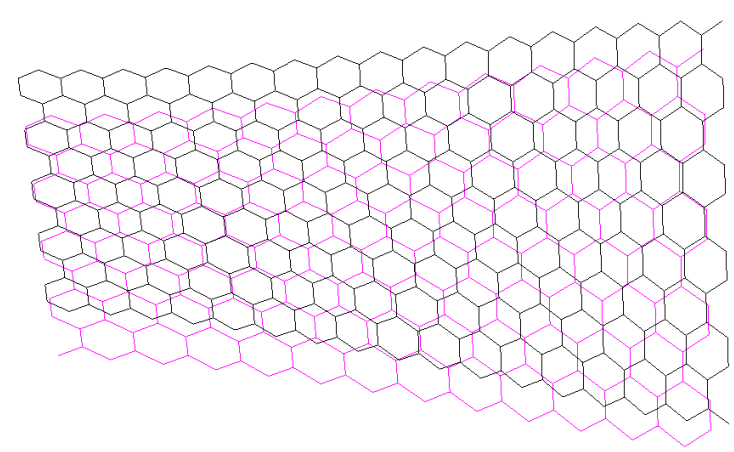

(c) First buckling mode shape of graphene-hBN hetero-structure

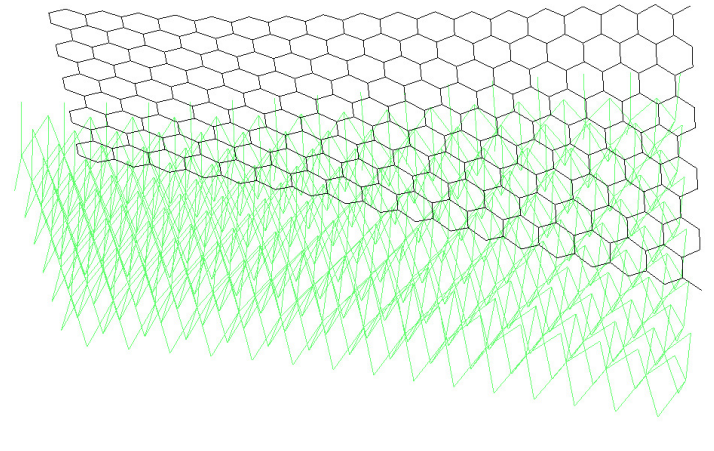

(d) First buckling mode shape of graphene- $\mathrm{MoS}_{2}$ hetero-structure

Figure 3: Cantilever boundary conditions and resulting buckling modes

\subsection{Dependence of buckling loads on length}

The variation of single layer critical buckling load with length is shown in Fig. 5. In order to understand the influence of the lattice mismatch on the buckling behavior of nanosheets, Fig. 6 plots the variation of the buckling capacity in double layered nano structures. We can observe here that the critical buckling load decreases when the sheet length increases. Such a variation is comparable with analytical calculations reported for elastic plates [34, 35]. For bridged single layer graphene with a length increasing from $2.3 \mathrm{~nm}$ to $20 \mathrm{~nm}$, the critical buckling load decreases from $6.2 \mathrm{~N}$ to $1.0 \mathrm{~N}$. Within the same length range, the bridged single layer $\mathrm{hBN}$ 


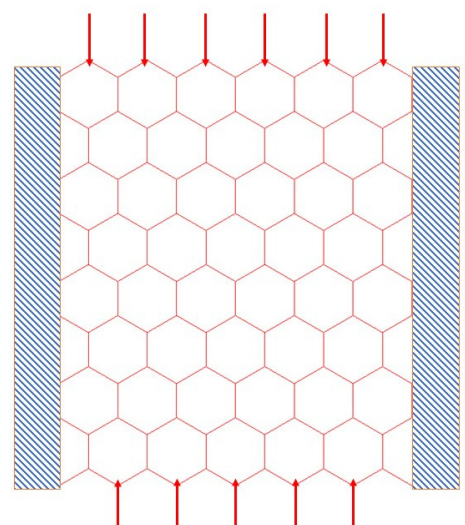

(a) Cantilever boundary condition

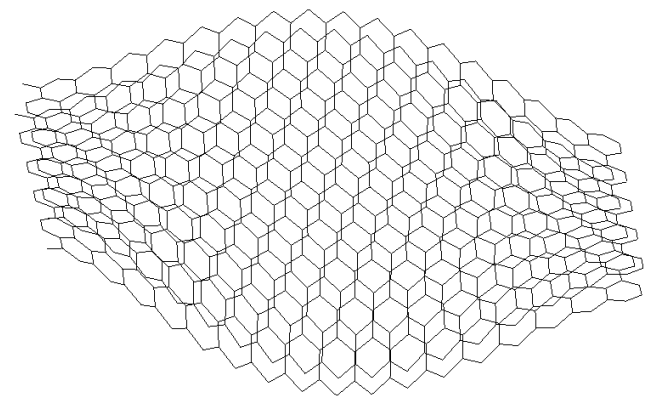

(b) First buckling mode shape of bilayer graphene

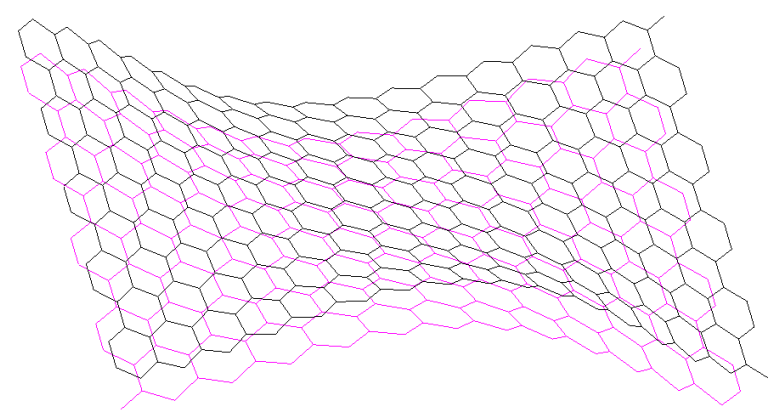

(c) First buckling mode shape of graphene-hBN hetero-structure

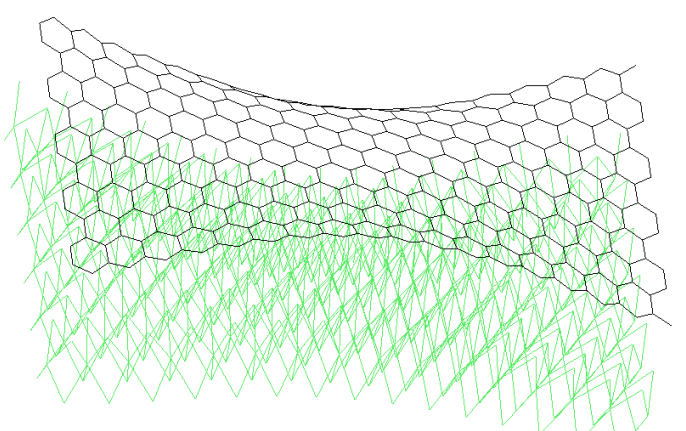

(d) First buckling mode shape of graphene- $\mathrm{MoS}_{2}$ hetero-structure

Figure 4: Bridged boundary conditions and resulting buckling modes

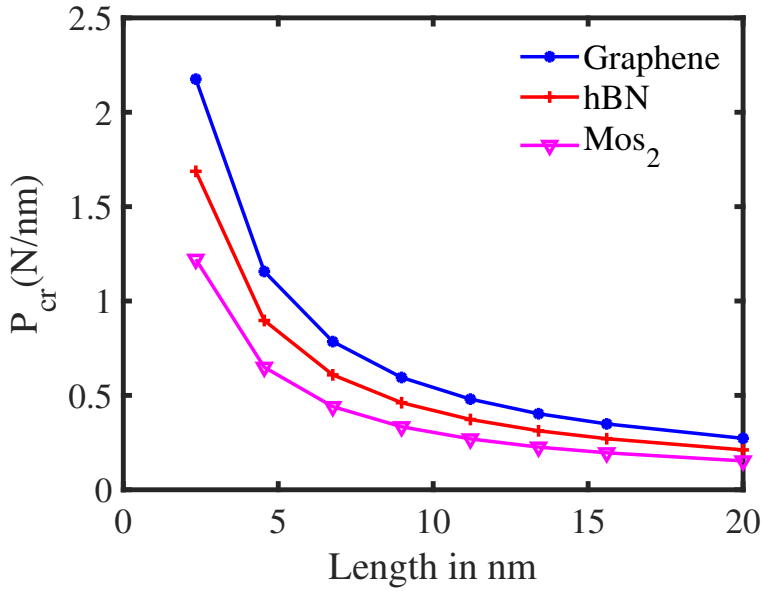

(a) Buckling loads - cantilevered single layer

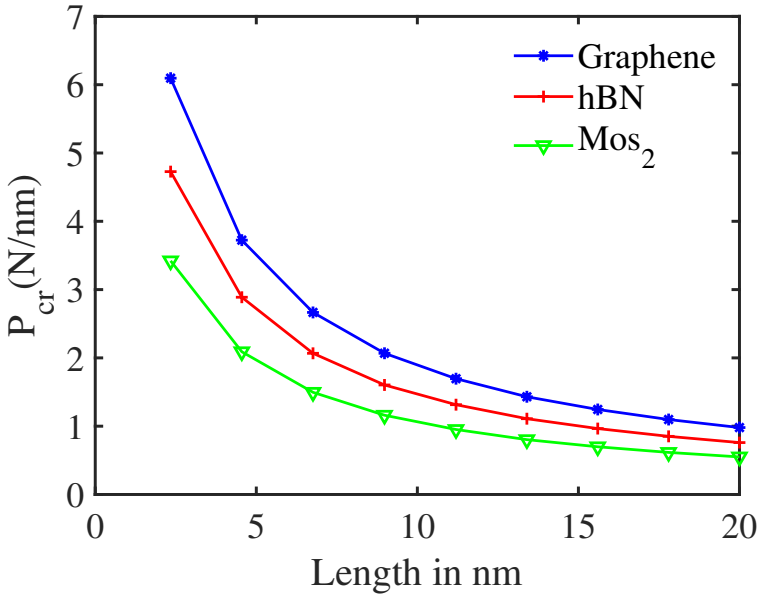

(b) Buckling loads - bridged single layer

Figure 5: Variation of buckling loads with length for single layers of graphene, hBN and $\mathrm{MoS}_{2}$. 
shows a decreasing critical buckling load from $4.7 \mathrm{~N}$ to $0.7 \mathrm{~N}$. For bridged single layer hBN, the critical buckling load decreases from $3.4 \mathrm{~N}$ to $0.5 \mathrm{~N}$. For bridged double layer graphene, the buckling load decreases from $179.3 \mathrm{~N}$ to $77.6 \mathrm{~N}$. For bridged graphene-hBN, the critical load decreases from $139.1 \mathrm{~N}$ to $60.2 \mathrm{~N}$, and for bridged graphene- $\mathrm{MoS}_{2}$, the buckling load decreases from 100.6 N to 43.6 N. From these observations, it can be concluded that, at a given length and under buckling loads, a double layer is stronger than the single layer system by a factor of more than 30. Among the three combinations considered here, namely graphene-graphene, graphene$\mathrm{hBN}$ and graphene- $\mathrm{MoS}_{2}$, the homogenous graphe-graphene offers the highest buckling strength for a given length. For instance, the buckling strength of the graphe-graphene combination is found to be $79 \%$ higher than that of graphene- $\mathrm{MoS}_{2}$, for the sheet size $2.3 \mathrm{~nm}$ x $9.4 \mathrm{~nm}$. The quantitative data showing buckling strength at various lengths, for each single and double layer type nanosheet are shown in Table 1 and Table 2. The data listed in these two tables corresponds to the bridged boundary condition. As per Fig. 5 and Fig. 6, the variation pattern of the critical buckling loads for double layer systems is found to be dissimilar to that of single layer system. Such a dissimilarity in pattern is due to the involvement of the interlayer L-J potential found in the double layer system.

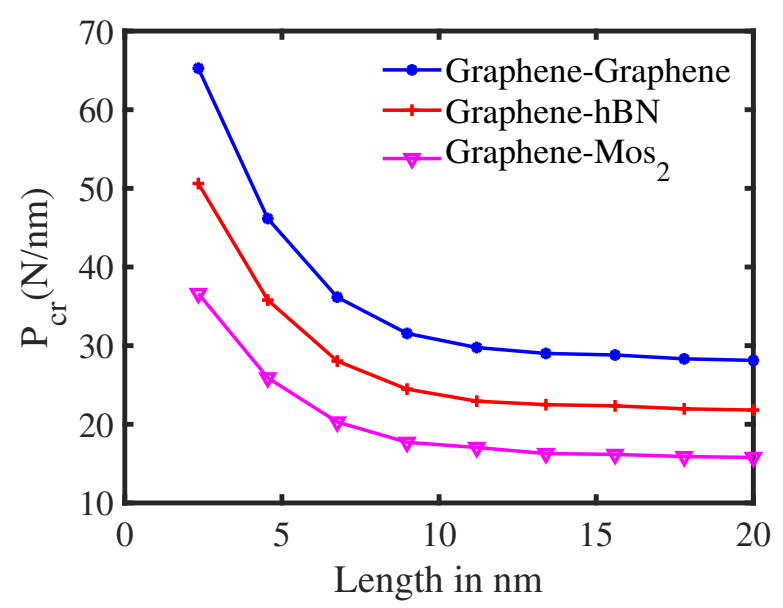

(a) Buckling loads - cantilevered double layer

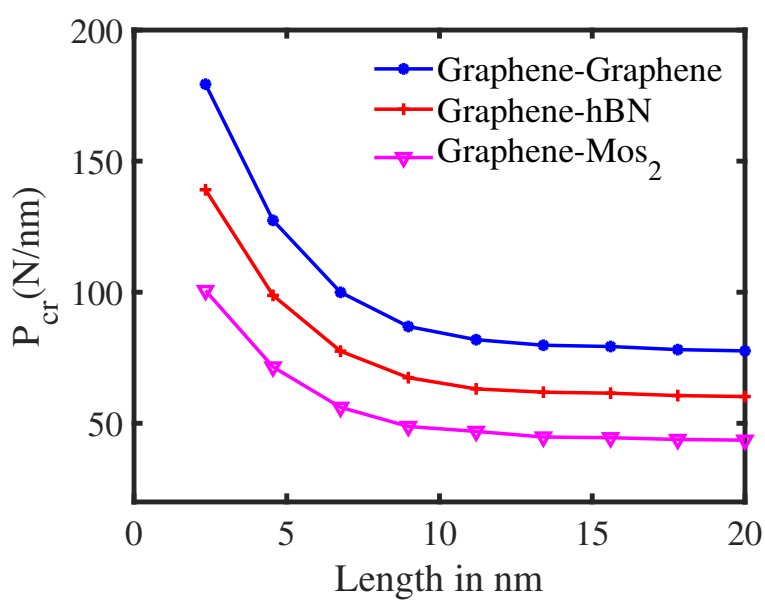

(b) Buckling loads - bridged double layer

Figure 6: Variation of buckling loads with length for double layer nanosheets of graphene-graphene, graphene-hBN and graphene- $\mathrm{MoS}_{2}$.

\subsection{Dependence of critical buckling load on boundary conditions}

From all the plots shown in the present work, it can be observed that the bridged nano structures offer higher buckling strength than that of cantilever nano structures, irrespective of the number of layers. As per the continuum mechanics of elastic plates [34, 35], a bridged 


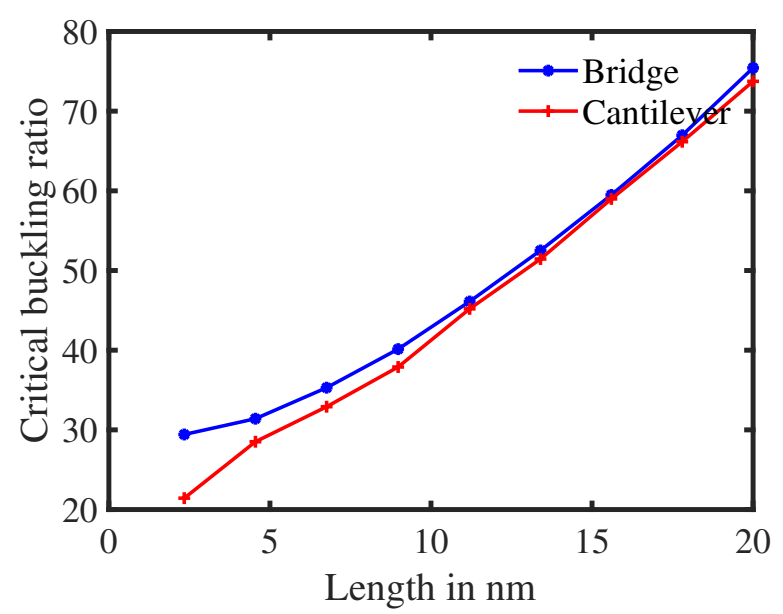

(a) The ratio: (Praphene-Graphene $\left./ P_{\text {Graphene }}\right)$

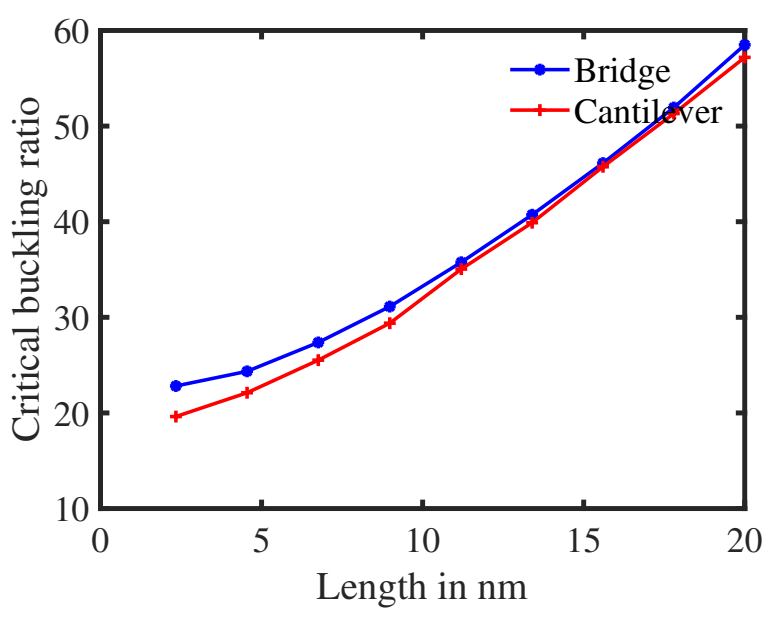

(b) The ratio: $P_{\text {Graphene-hBN }} / P_{\text {Graphene }}$

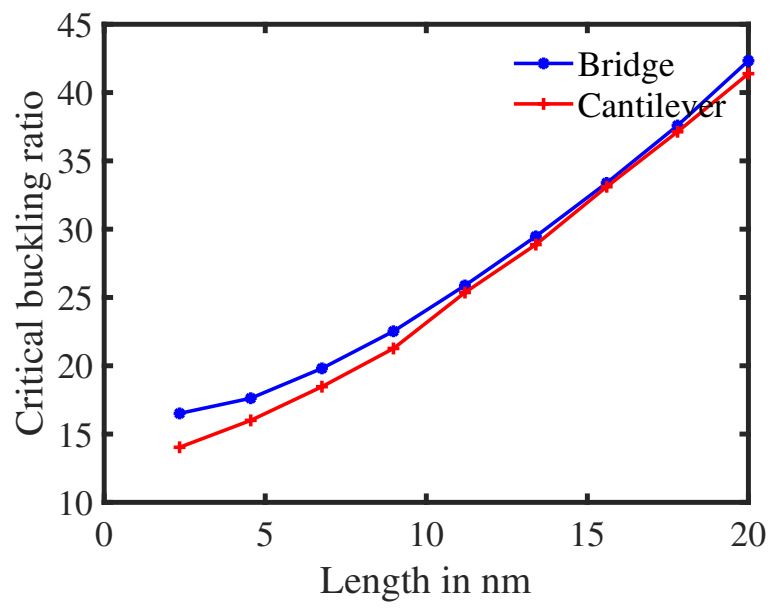

(c) The ratio $: P_{\text {Graphene-MoS }} / P_{\text {Graphene }}$

Figure 7: Comparison between single layer graphene and double layer combinations.

plate structure offers higher stiffness as compared to a cantilever structure. Therefore, a bridged elastic plate or sheet will be stronger under buckling as compared to a cantilevered plate or sheet. A comparison between the bridged and cantilevered nanosheets can be found in the plots shown in Fig. 5. These plots indicate that by changing the boundary condition from cantilever to bridge type the critical buckling load increases up to about three times in the case of single layer graphene. Similar level of enhancement is also observed for single layers of $\mathrm{hBN}$ and $\mathrm{MoS}_{2}$. For double layer systems (Fig. 6), the trend is similar. For single layer sheet models, the slope observed in the curves of cantilever and bridge sheets is found to be identical at lower lengths $(<10 \mathrm{~nm})$. Whereas for double layer sheets, the slope of the curves of cantilever sheets is found to be higher as compared to the slope of bridged sheet curves, at lower lengths $(<10 \mathrm{~nm})$. Such a discrepancy is the result of the interlayer L-J potential present in the double layer system. 


\subsection{Enhancement in buckling capacity of graphene sheet due to the addition of another graphene, $h B N$ or $M_{0} S_{2}$ sheet}

The variation of the ratio between the critical buckling loads of the double ( $\left.P_{\text {Graphene-Graphene }}\right)$ and single layer graphene $\left(P_{\text {Graphene }}\right)$ is given in Fig.7(a). The buckling load ratio between the combined graphene-hBN sheet $\left(P_{\text {Graphene-hBN }}\right)$ and the single layer graphene $\left(P_{\text {Graphene }}\right)$ is given in Fig.7(b). For the combined graphene- $\mathrm{MoS}_{2}$ sheet $\left(P_{\text {Graphene-MoS }}\right)$ and the single layer graphene $\left(P_{\text {Graphene }}\right)$, the critical load ratio is given in Fig. $7(\mathrm{c})$. Since the ratio remains higher than one in all the plots, it can be concluded that the addition of another layer (of any of the three nanomaterials studied here), always results in an increase in the buckling strength of the graphene sheet. As per the plots (Fig. 7) and sheet sizes considered in the current work, it is possible to observe that the addition of a nanosheet on top of the graphene sheet, leading to a lattice mismatch, results in a buckling strength increase up to 75 times. Such a great enhancement is due to the additional graphene nanosheet. If the additional sheet is of $\mathrm{hBN}$ or $\mathrm{MoS}_{2}$ type, then the enhancement in buckling strength can be up to 59 times or 43 times, respectively.

The addition of another layer of a nanosheet to a graphene sheet results in higher buckling strength as compared to that of a graphene sheet alone. In order to quantify such an enhancement, buckling strength data is presented in Table 1 and Table 2. Table 1 shows values of buckling strength of single layers of graphene, $\mathrm{hBN}$ and $\mathrm{MoS}_{2}$ for various lengths, under bridged boundary conditions. As per this table, single layer graphene offers the highest buckling strength at a given length, as compared to that of single layers of $\mathrm{hBN}$ and $\mathrm{MoS}_{2}$. Among the three single layer nanosheets considered, $\mathrm{MoS}_{2}$ offers the least buckling strength. Table 2 shows values of buckling strength of graphene-graphene, graphene-hBN and graphene-MoS $\mathrm{M}_{2}$ double layer nanosheets at various lengths and under bridged boundary conditions. This table proves that for a graphene sheet, the addition of another graphene sheet offers the highest buckling strength when compared to the addition of $\mathrm{hBN}$ or $\mathrm{MoS}_{2}$ sheets. The inclusion of further layers leading to a three- or four-layer system, further enhances the buckling strength of the nano structure. As compared to the bilayer graphene presented in the literature [2], the current configuration with $1.1^{\circ}$ interlayer twist is found to be offering slightly lower $(\sim 2 \%)$ buckling capacity at a given sheet length.

Table 1 and Table 2 indicate that the variation of the single layer critical load is found to be identical with that of double layered sheets(i.e. decreasing with length). However, there is an observable difference in slopes. The slope for single layer graphene is found to be higher in regards to single layer hBN and $\mathrm{MoS}_{2}$. The slope for double layer graphene is found to be higher 
in regards to graphene-hBN and graphene- $\mathrm{MoS}_{2}$.

Table 1: Buckling load capacities for single layer graphene, $\mathrm{hBN}$ and $\mathrm{MoS}_{2}$. The single layer sheets considered here are bridged. Graphene offers higher buckling strength among the three types of nanosheets considered.

\begin{tabular}{cccc}
\hline Length $(\mathrm{nm})$ & $P_{\text {Graphene }}(\mathrm{N})$ & $P_{h B N}(\mathrm{~N})$ & $P_{M o S_{2}}(\mathrm{~N})$ \\
\hline 2.3 & 6.2 & 4.7 & 3.4 \\
4.5 & 4.1 & 2.9 & 2.1 \\
6.7 & 2.9 & 2.1 & 1.5 \\
8.9 & 2.2 & 1.61 & 1.2 \\
11.2 & 1.9 & 1.3 & 0.9 \\
13.4 & 1.6 & 1.1 & 0.8 \\
15.6 & 1.4 & 1.0 & 0.7 \\
17.8 & 1.2 & 0.8 & 0.6 \\
20.0 & 1.1 & 0.7 & 0.5 \\
\hline
\end{tabular}

Table 2: Comparison of bilayer graphene buckling loads against graphene-hBN and graphene-MoS 2 buckling loads. The boundary condition considered here is the bridged type. Graphene-graphene offers higher buckling strength among the three different combinations of double layers.

\begin{tabular}{cccc}
\hline Length $(\mathrm{nm})$ & $P_{\text {Graphene-Graphene }}(\mathrm{N})$ & $P_{\text {Graphene-hBN }}(\mathrm{N})$ & $P_{\text {Graphene-MoS }}(\mathrm{N})$ \\
\hline 2.3 & 179.3 & 139.1 & 100.6 \\
4.5 & 127.4 & 98.8 & 71.5 \\
6.7 & 99.9 & 77.5 & 56.1 \\
8.9 & 86.9 & 67.4 & 48.8 \\
11.2 & 81.8 & 63.1 & 46.9 \\
13.4 & 79.7 & 61.9 & 44.8 \\
15.6 & 79.3 & 61.5 & 44.5 \\
17.8 & 78.1 & 60.6 & 43.8 \\
20.0 & 77.6 & 60.2 & 43.6 \\
\hline
\end{tabular}

\section{Conclusions}

The buckling strength of homogeneous and heterogenous carbon based nano structures has been investigated by means of an atomistic finite element approach. In particular, homogeneous graphene-graphene along with heterogeneous graphene-hBN and graphene- $\mathrm{MoS}_{2}$ nanosheets have been studied. Lattice mismatch was present in all of the double layer configurations considered in the current study. Lattice mismatch was attained in graphene-graphene sheets by aligning the sheets at an angle of $1.1^{\circ}$. For the case of bilayer graphene, the introduction of such a twist angle has led to a slight decrease in buckling strength. This investigation confirmed that the bridged nanosheets offer higher buckling strength as compared to that of cantilevered nanosheets. For all of the nanosheet types studied, the critical buckling load reduced for a length increase. The addition of nanosheets such as graphene, $\mathrm{hBN}$ and $\mathrm{MoS}_{2}$ on top of a single layer 
graphene sheet led to a significant increase in buckling strength. Among the three types of nano materials considered here as the addition, namely graphene, $\mathrm{hBN}$ and $\mathrm{MoS}_{2}$, graphene showed the highest buckling strength. The current analysis considered lattice mismatched double layers of nanosheets. It is estimated that the involvement of more layers can further enhance the buckling strength.

\section{References}

[1] K. S. Novoselov, A. K. Geim, S. V. Morozov, D. Jiang, Y. Zhang, S. V. Dubonos, I. V. Grigorieva, A. A. Firsov, Electric field effect in atomically thin carbon films, Science 306 (5696) (2004) 666-669. arXiv:https://science.sciencemag.org/content/306/5696/666.full.pdf, doi:10.1126/science.1102896.

URL https://science.sciencemag.org/content/306/5696/666

[2] Y. Chandra, R. Chowdhury, S. Adhikari, F. Scarpa, Elastic instability of bilayer graphene using atomistic finite element, Physica E: Low-dimensional Systems and Nanostructures 44 (1) (2011) 12 - 16. doi:https://doi.org/10.1016/j.physe.2011.06.020.

URL http://www.sciencedirect.com/science/article/pii/S1386947711002232

[3] Y. Chandra, E. I. Saavedra Flores, F. Scarpa, S. Adhikari, Buckling of hybrid nano composites with embedded graphene and carbon nanotubes, Physica E 83 (2016) 434-441. doi:http://dx.doi. org/10.1016/j.physe.2016.01.021.

[4] L. Song, L. Ci, H. Lu, P. B. Sorokin, C. Jin, J. Ni, A. G. Kvashnin, D. G. Kvashnin, J. Lou, B. I. Yakobson, P. M. Ajayan, Large scale growth and characterization of atomic hexagonal boron nitride layers, Nano Letters 10 (8) (2010) 3209-3215, pMID: 20698639. arXiv:https://doi .org/10.1021/ nl1022139, doi:10.1021/nl1022139.

URL https://doi.org/10.1021/nl1022139

[5] V. Barone, J. E. Peralta, Magnetic boron nitride nanoribbons with tunable electronic properties, Nano Letters 8 (8) (2008) 2210-2214, pMID: 18624385. arXiv:https://doi.org/10.1021/ nl080745j, doi:10.1021/n1080745j.

URL https://doi.org/10.1021/nl080745j

[6] Z. He, W. Que, Molybdenum disulfide nanomaterials: Structures, properties, synthesis and recent progress on hydrogen evolution reaction, Applied Materials Today 3 (2016) 23 - 56. doi:https: //doi.org/10.1016/j.apmt.2016.02.001.

URL http://www.sciencedirect.com/science/article/pii/S2352940716300014

[7] S. Najmaei, M. Amani, M. L. Chin, Z. Liu, A. G. Birdwell, T. P. ORegan, P. M. Ajayan, M. Dubey, J. Lou, Electrical transport properties of polycrystalline monolayer molybdenum disulfide, ACS Nano 8 (8) (2014) 7930-7937, pMID: 25019978. arXiv:https://doi.org/10.1021/nn501701a, doi:10.1021/nn501701a.

URL https://doi.org/10.1021/nn501701a

[8] Y. Gao, Q. Liu, B. Xu, Lattice mismatch dominant yet mechanically tunable thermal conductivity in bilayer heterostructures, ACS Nano 10 (5) (2016) 5431-5439, pMID: 27093571. arXiv:https: //doi.org/10.1021/acsnano.6b01674, doi:10.1021/acsnano.6b01674.

URL https://doi.org/10.1021/acsnano.6b01674

[9] S. Bertolazzi, J. Brivio, A. Kis, Stretching and breaking of ultrathin mos2, ACS Nano 5 (12) (2011) 9703-9709, pMID: 22087740. arXiv:https://doi.org/10.1021/nn203879f, doi:10.1021/ nn203879f.

URL https://doi.org/10.1021/nn203879f

[10] L. Wang, S. Zihlmann, M.-H. Liu, P. Makk, K. Watanabe, T. Taniguchi, A. Baumgartner, C. Schonenberger, New generation of moire superlattices in doubly aligned hbn/graphene/hbn heterostructures, Nano Letters 19 (4) (2019) 2371-2376.

[11] D. L. Nika, A. I. Cocemasov, A. A. Balandin, Specific heat of twisted bilayer graphene: Engineering phonons by atomic plane rotations, Applied Physics Letters 105 (3) (2014) 031904. arXiv:https: 
//doi.org/10.1063/1.4890622, doi:10.1063/1.4890622.

URL https://doi.org/10.1063/1.4890622

[12] B. Mortazavi, G. R. Berdiyorov, M. Makaremi, T. Rabczuk, Mechanical responses of two-dimensional mote2; pristine 2h, $1 \mathrm{t}$ and $1 \mathrm{t}$ and 1t/2h heterostructure, Extreme Mechanics Letters 20 (2018) 65 72. doi:https://doi.org/10.1016/j.eml.2018.01.005.

URL http://www.sciencedirect.com/science/article/pii/S2352431617302213

[13] J. E. Barrios-Vargas, B. Mortazavi, A. W. Cummings, R. Martinez-Gordillo, M. Pruneda, L. Colombo, T. Rabczuk, S. Roche, Electrical and thermal transport in coplanar polycrystalline graphenehbn heterostructures, Nano Letters 17 (3) (2017) 1660-1664, pMID: 28195494. arXiv: https://doi.org/10.1021/acs.nanolett.6b04936, doi:10.1021/acs.nanolett.6b04936.

URL https://doi.org/10.1021/acs.nanolett.6b04936

[14] E. I. Saavedra Flores, S. Adhikari, M. Friswell, F. Scarpa, Hyperelastic axial buckling of single wall carbon nanotubes, Physica E: Low-dimensional Systems and Nanostructures 44 (2) (2011) 525 529. doi:https://doi.org/10.1016/j.physe.2011.10.006.

URL http://www.sciencedirect.com/science/article/pii/S1386947711003687

[15] Y. Chandra, R. Chowdhury, F. Scarpa, S. Adhikari, Vibrational characteristics of bilayer graphene sheets, Thin Solid Films 519 (18) (2011) 6026-6032. doi:https://doi.org/10.1016/j.tsf.2011. 04.012 .

URL http://www.sciencedirect.com/science/article/pii/S0040609011008248

[16] Y. Chandra, R. Chowdhury, F. Scarpa, S. Adhikari, J. Sienz, C. Arnold, T. Murmu, D. Boulda, Vibration frequency of graphene based composites: A multiscale approach, Materials Science and Engineering B 177 (2012) 303 310. doi:http://dx.doi.org/10.1016/j.mseb.2011.12.024.

[17] Y. Chandra, F. Scarpa, R. Chowdhury, S. Adhikari, J. Sienz, Multiscale hybrid atomistic-fe approach for the nonlinear tensile behaviour of graphene nanocomposites, Composites Part A 46 (2013) 147153. doi:https://doi.org/10.1016/j.compositesa.2012.11.006.

[18] Y. Chandra, F. Scarpa, S. Adhikari, J. Zhang, E. I. Saavedra Flores, H. Peng, Pullout strength of graphene and carbon nanotube/epoxy composites, Composites Part B 102 (1-8). doi:http: //dx.doi.org/10.1016/j.compositesb.2016.06.070.

[19] T. Mukhopadhyay, A. Mahata, S. Adhikari, M. A. Zaeem, Effective elastic properties of two dimensional multiplanar hexagonal nanostructures, 2D Materials 4 (2) (2017) 025006. doi:10.1088/ 2053-1583/aa551c.

URL https://doi.org/10.1088\%2F2053-1583\%2Faa551c

[20] C. Li, T.-W. Chou, A structural mechanics approach for the analysis of carbon nanotubes, International Journal of Solids and Structures 40 (10) (2003) 2487 - 2499. doi:https://doi.org/10. 1016/S0020-7683(03)00056-8.

URL http://www.sciencedirect.com/science/article/pii/S0020768303000568

[21] F. Scarpa, S. Adhikari, R. Chowdhury, The transverse elasticity of bilayer graphene, Physics Letters A 374 (19) (2010) 2053 - 2057. doi:https://doi.org/10.1016/j.physleta.2010.02.063. URL http://www.sciencedirect.com/science/article/pii/S0375960110002240

[22] L. A. Girifalco, M. Hodak, R. S. Lee, Carbon nanotubes, buckyballs, ropes, and a universal graphitic potential, Phys. Rev. B 62 (2000) 13104-13110. doi:10.1103/PhysRevB.62.13104. URL https://link.aps.org/doi/10.1103/PhysRevB.62.13104

[23] J.-W. Jiang, H. S. Park, Mechanical properties of mos2/graphene heterostructures, Applied Physics Letters 105 (3) (2014) 033108. arXiv:https://doi.org/10.1063/1.4891342, doi:10.1063/1. 4891342.

URL https://doi.org/10.1063/1.4891342

[24] R. M. Elder, M. R. Neupane, T. L. Chantawansri, Stacking order dependent mechanical properties of graphene/mos2 bilayer and trilayer heterostructures, Applied Physics Letters 107 (7) (2015) 073101. arXiv:https://doi.org/10.1063/1.4928752, doi:10.1063/1.4928752.

URL https://doi.org/10.1063/1.4928752

[25] A. J. Pak, G. S. Hwang, Theoretical analysis of thermal transport in graphene supported on hexagonal boron nitride: The importance of strong adhesion due to $\pi$-bond polarization, Phys. Rev. Applied 6 (2016) 034015. doi:10.1103/PhysRevApplied.6.034015.

URL https://link.aps.org/doi/10.1103/PhysRevApplied.6.034015 
[26] M. Neek-Amal, F. M. Peeters, Graphene on boron-nitride: Moir pattern in the van der waals energy, Applied Physics Letters 104 (4) (2014) 041909. arXiv: https://doi.org/10.1063/1.4863661, doi: 10.1063/1.4863661.

URL https://doi.org/10.1063/1.4863661

[27] F. Scarpa, S. Adhikari, A mechanical equivalence for poisson's ratio and thickness of cc bonds in single wall carbon nanotubes, Journal of Physics D: Applied Physics 41 (8) (2008) 085306.

URL http://stacks.iop.org/0022-3727/41/i=8/a=085306

[28] F. Scarpa, S. Adhikari, Uncertainty modeling of carbon nanotube terahertz oscillators, Journal of Non-Crystalline Solids 354 (35) (2008) 4151 - 4156, functional and Nanostructured Materials. doi:https://doi.org/10.1016/j.jnoncrysol.2008.06.065.

URL http://www.sciencedirect.com/science/article/pii/S0022309308003840

[29] K. Chenoweth, A. C. T. van Duin, W. A. Goddard, Reaxff reactive force field for molecular dynamics simulations of hydrocarbon oxidation, The Journal of Physical Chemistry A 112 (5) (2008) 10401053.

[30] Y. Cao, V. Fatemi, S. Fang, K. Watanabe, T. Taniguchi, E. Kaxiras, P. Jarillo-Herrero, Unconventional superconductivity in magic-angle graphene superlattices, Nature 556 (7699) (2018) 4350. doi:10.1038/nature26160.

URL https://doi.org/10.1038/nature26160

[31] R. Bistritzer, A. H. MacDonald, Moiré bands in twisted double-layer graphene, Proceedings of the National Academy of Sciences 108 (30) (2011) 12233-12237. arXiv:https://www.pnas.org/ content/108/30/12233.full.pdf, doi:10.1073/pnas.1108174108.

URL https://www. pnas.org/content/108/30/12233

[32] S. Shallcross, S. Sharma, O. A. Pankratov, Quantum interference at the twist boundary in graphene, Phys. Rev. Lett. 101 (2008) 056803. doi:10.1103/PhysRevLett.101.056803.

URL https://link.aps.org/doi/10.1103/PhysRevLett.101.056803

[33] Y. Cao, V. Fatemi, A. Demir, S. Fang, S. L. Tomarken, J. Luo, J. D. Sanchez-Yamagishi, K. Watanabe, T. Taniguchi, E. Kaxiras, R. Ashoori, P. Jarillo-Herrero, Correlated insulator behaviour at half-filling in magic-angle graphene superlattices, Nature 556 (2018) 80-84.

[34] S. P. Timoshenko, Theory of Plates and Shells, McGraw-Hill, New York, 1940.

[35] S. P. Timoshenko, J. M. Gere, Theory of elastic stability, McGraw-Hill, New York, 1961. 